\title{
Raman spectroscopy of individual poly(ethylene oxide) electrospun fibers: Effect of the collector on molecular orientation
}

\author{
Marie Richard-Lacroix* and Christian Pellerin \\ Département de chimie and Centre for Self-Assembled Chemical Structures \\ Université de Montréal, Montréal, QC, H3C 3J7, Canada \\ * Corresponding author: Tel.: (514) 340-5762; fax: (514) 340-5290; \\ Emails: marie.richard-lacroix@umontreal.ca; c.pellerin@umontreal.ca
}




\begin{abstract}
.
Electrospun fibers are complex polymeric materials with a typical diameter of a few hundred nanometers that are often frozen in out-of-equilibrium states due to the fast solvent evaporation and high elongational forces during the process. Their molecular orientation, and the parameters affecting it, is a critical (although challenging) parameter to characterize because it allows improving and tuning many properties. In this paper, we characterize molecular orientation of poly(ethylene oxide) (PEO) fibers produced with the three most common collectors by applying our recently developed Raman spectroscopy methodologies to probe individual fibers. Results reveal an extremely high orientation in all PEO fibers and, surprisingly, the absence of any effect of the collector on molecular orientation. This contrasts with reports by several other research groups in the context of studies on bundles of fibers, as well as with our WAXD results on the same fibers. It is concluded that studies on bundles composed of thousands of imperfectly aligned fibers lead to a systematic underestimation of the orientation and to apparent results that prevent us from drawing clear conclusions on the molecular impact of processing parameters when they generate different levels of macroscopic alignment. This work highlights how Raman spectroscopy helps to overcome one of the major challenges in the field, the characterization of individual fibers, to reach a better understanding and control of electrospun fibers properties.
\end{abstract}

Keywords. Confocal Raman spectroscopy, molecular orientation, fibers, electrospinning. 


\section{Introduction.}

Electrospinning is a widely used technique for producing continuous fibers through the application of a high voltage on an entangled polymer solution. The resulting fibers have a typical diameter of a few hundred $\mathrm{nm}$ and thus show an extremely large area/volume ratio that makes them useful in domains such as tissue engineering or selective filtration.[15] Recently, they also showed excellent performance when integrated in optical and electronic devices such as organic field effect transistors (OFET)[6-8] and polymer lightemitting diodes (PLED).[9,10]

One of the drawbacks of electrospun fibers that limits their widespread application is the difficulty of establishing their structure/properties relationships and identifying the experimental factors affecting properties, limiting our capacity to modulate them.[11] Molecular orientation is an important parameter because it enhances many properties (for instance the elastic modulus and the electrical or thermal conductivity) along the main axis. One of the factors suspected of influencing the molecular orientation of fibers is the type of collector on which they are deposited at the last stage of their formation. In fact, molecular orientation in electrospun fibers was historically considered to originate mainly from the additional stretching induced by the collector and/or to its effect on the electric field that, in one way or another, promotes stretching of the polymer chains.[12-14] A myriad of types of collectors and modified electrospinning setups have been developed with the aim, among others, to increase the control over fiber alignment[15-17] (which is necessary for many applications such as tissue engineering and drug release[18,19]) or to enable highly targeted deposition.[20-22] Electrospun fibers are most commonly collected 
on a simple plate such as an aluminum foil, on two metallic rods separated by a small gap (a 2 rods collector), or on a rotating disk.

Due to their intrinsically small size, electrospun fibers must often be studied as bundles composed of thousands of fibers in order to obtain a satisfying signal to noise ratio when using conventional characterization techniques such as infrared spectroscopy and wide angle X-ray diffraction. The orientation measured on such bundles depends not only on the molecular orientation of the polymer chains within each fiber but also on the macroscopic alignment of the fibers. Since the collector type can affect both parameters, its real impact remains largely misunderstood. Accordingly, strategies had to be developed in order to evaluate both parameters separately. The most common is to collect samples with a similar macroscopic organization to evaluate more specifically the effect of the different collectors on molecular orientation.[23] The other is to quantify the macroscopic alignment of the fibers and to take it into account in the calculation of molecular orientation for fibers prepared under different collection conditions.[24,25] In all cases, a significant impact on orientation has been reported for the parameters that promote fiber alignment, such as the increase of the collector rotation speed or the increase of the distance between the two metallic rods.

In recent years, the development of methodologies enabling the study of individual electrospun fibers has lifted a veil on several of their interesting characteristics, in particular an increase of modulus when reducing the fiber diameter[11] that has been associated to an increase of the level of molecular orientation. The most common technique to characterize orientation at the individual fiber level is selected area electron diffraction (SAED), which gives access to a resolution of a few tens of nanometers.[23,26-28] It is 
however limited to the crystalline phase and requires extensive sample preparation, such that SAED studies have been limited to a small number of fibers and to only a few parameters (including some collector aspects). $[23,26]$ In a recent series of publications, we have demonstrated that confocal Raman spectroscopy is an efficient tool for the in-depth characterization of electrospun fibers.[29,30] It provides molecular level specificity and allows detailed analysis with a submicron resolution that is on the order of the typical diameter of an electrospun fiber. Its application to a wide variety of systems was facilitated by the development of a new methodology that simplifies the experimental procedure for orientation quantification and gives access to more information than the conventional method.[31,32] For instance, by studying large quantities of polystyrene fibers, we have established the first structure/properties relationship at the individual fiber level by correlating the molecular orientation and the mechanical properties of individual fibers.[30]

In this publication, we use polarized Raman spectroscopy to characterize the effect of the collector on the molecular orientation of individual electrospun fibers of poly(ethylene oxide) (PEO). Samples were produced using the three most common collection modes in order to discriminate their impacts on the macroscopic organization of 
the fibers and on their microstructure. Our results reveal the unexpected absence of any collector effect on molecular orientation, which is extremely high in all cases.

\section{Experimental section.}

Electrospinning. Poly(ethylene oxide) (Scientific Polymer Products) with a weightaverage molecular weight of $400 \mathrm{~kg} / \mathrm{mol}$ and methanol (Fisher Scientific) were used as received. Fibers were prepared by dissolving $0.6 \mathrm{~g}$ of PEO in $10 \mathrm{ml}$ of methanol $(6 \% \mathrm{w} / \mathrm{v})$ and placing the solution in a glass syringe equipped with a $0.41 \mathrm{~mm}$ diameter flat-end needle. A PHD 2000 syringe pump (Harvard Apparatus) was used to impose a constant flow of $0.02 \mathrm{ml} / \mathrm{h}$. A $15 \mathrm{kV}$ positive voltage was applied to the needle tip using a CZE 1000R high-voltage power supply (Spellman High Voltage Electronics). Three types of collector were used for fibers deposition, namely, a rotating metallic disk, a 2 rods collector (with the rods being separated by $20 \mathrm{~mm}$ ) and an aluminum foil, that acted as a counter electrode on which a negative $2 \mathrm{kV}$ potential (Power Designs) was applied. The distance between the needle tip and the collector was $15 \mathrm{~cm}$ in all cases. For Raman spectroscopy experiments, small quantities of fibers were carefully transferred on $\mathrm{BaF}_{2}$ windows and dried under vacuum for at least $24 \mathrm{~h}$ prior to analysis.

Scanning electron microscopy. Field-emission scanning electron microscopy (SEM) images were recorded with a JEOL JSM-7400F instrument operated at a voltage of $1.5 \mathrm{kV}$ 
and a current of $20 \mu \mathrm{A}$ under a high vacuum. A thin $(\sim 5 \mathrm{~nm})$ gold film was deposited on the fibers prior to imaging.

Wide angle X-ray diffraction. WAXD measurements were recorded by 1 hour acquisitions for all samples using a Bruker AXS diffractometer (Siemens Kristalloflex 780 generator) with a $\mathrm{CuK}_{\alpha}$ source $(\lambda=0.1542 \mathrm{~nm})$ collimated to $\sim 400 \mu \mathrm{m}$ with a graphite monochromator and a two dimensional detector (HI-STAR). A 1 hour background was subtracted from all 2D patterns recorded. Oriented fiber bundles were placed in a homemade sample holder such that the fibers long axis was oriented vertically.

Raman spectroscopy. Spectra were recorded in the backscattering geometry using the $632.8 \mathrm{~nm}$ He-Ne laser of a LabRam HR800 spectrometer (Horiba Scientific) coupled with an Olympus BX41 microscope and with use of a long working distance $100 \mathrm{X}$ objective $(\mathrm{NA}=0.8)$. The confocal hole and the slit were fixed at 100 and $150 \mu \mathrm{m}$, respectively. The detailed experimental procedure for Raman measurements on individual electrospun fibers is provided elsewhere.[29] The polarization of the laser and of the Raman scattering, parallel $(\mathrm{Z})$ and perpendicular $(\mathrm{X})$ to the fiber axis, were selected with the help of a halfwave plate and a polarizer, respectively. A scrambler was inserted in the optical path before the 600 grooves/mm holographic grating to minimize its polarization dependence. The residual polarization differences were corrected with help of an isotropic sample, as described elsewhere.[29] The integration time for each spectrum was fixed between 10 and $15 \mathrm{~s}$, averaged 10 to 15 times.

\section{Theoretical section.}


The molecular orientation for any system showing a uniaxial symmetry can be described by the orientation distribution function $(N(\theta))$ which is an expansion of even Legendre polynomials $\left(P_{l}(\cos \theta)\right)$.[33]

$N(\theta)=\sum_{l}^{\text {even }}\left(l+\frac{1}{2}\right)\left\langle P_{l}\right\rangle P_{l}(\cos \theta)$

The $\left\langle P_{l}\right\rangle$ coefficients are determined experimentally and used to describe the level of orientation in the system. The $\left\langle P_{2}\right\rangle$ value (Eq. 2a) ranges between 0 (isotropic distribution of the units) and 1 (perfect orientation of the units along the fiber axis) for parallel orientations. A perfect orientation perpendicular to the main axis is associated with a $\left\langle P_{2}\right\rangle$ value of -0.5 . The $\left\langle P_{4}\right\rangle$ order parameter (Eq. 2b) enables polishing the shape of the orientation distribution and can reach limiting values that depend on $\left\langle P_{2}\right\rangle .[33,34]$

$\left\langle P_{2}\right\rangle=\frac{\left\langle 3 \cos ^{2} \theta-1\right\rangle}{2}$

$\left\langle P_{4}\right\rangle=\frac{\left\langle 35 \cos ^{4} \theta-30 \cos ^{2} \theta+3\right\rangle}{8}$

Polarized Raman intensities are related to quadratic summations on all units of the Raman tensor components $\left(\left\langle\left(\alpha_{i j}\right)^{2}\right\rangle\right)$.[35] In the backscattering geometry, these equations are expressed as linear functions of the orientation parameters $\left(\left\langle P_{2}\right\rangle\right.$ and $\left.\left\langle P_{4}\right\rangle\right)$, of the elements of the Raman tensor $(a)$ with the approximation that the tensor is cylindrical, and of a constant (b) that depends on the Raman tensor and on instrumental parameters such as the laser intensity.[36] 


$$
\begin{aligned}
& \left\langle\left(\alpha_{Z Z}\right)^{2}\right\rangle=b\left(\frac{1}{15}\left(3+4 a+8 a^{2}\right)+\frac{4}{21}\left(3+a-4 a^{2}\right)\left\langle P_{2}\right\rangle+\frac{8}{35}(1-a)^{2}\left\langle P_{4}\right\rangle\right) \\
& \left\langle\left(\alpha_{X X}\right)^{2}\right\rangle=b\left(\frac{1}{15}\left(3+4 a+8 a^{2}\right)-\frac{2}{21}\left(3+a-4 a^{2}\right)\left\langle P_{2}\right\rangle+\frac{3}{35}(1-a)^{2}\left\langle P_{4}\right\rangle\right) \\
& \left\langle\left(\alpha_{X Z}\right)^{2}\right\rangle=\left\langle\left(\alpha_{Z X}\right)^{2}\right\rangle=\left\langle\left(\alpha_{Z Y}\right)^{2}\right\rangle=b(1-a)^{2}\left(\frac{1}{15}+\frac{1}{21}\left\langle P_{2}\right\rangle-\frac{4}{35}\left\langle P_{4}\right\rangle\right) \\
& \left\langle\left(\alpha_{X Y}\right)^{2}\right\rangle=b(1-a)^{2}\left(\frac{1}{15}-\frac{2}{21}\left\langle P_{2}\right\rangle+\frac{1}{35}\left\langle P_{4}\right\rangle\right)
\end{aligned}
$$

In the backscattering geometry, we have access to 4 polarized spectra: 2 parallel-polarized (ZZ and $\mathrm{XX}$ ) and two cross-polarized (ZX and $\mathrm{XZ}$ ) spectra, where the first letter corresponds to the polarization of the incident laser and the second to the polarization of the scattered beam, as represented in Scheme 1.

\section{[Figure 1 should be here]}

By ratioing the polarized spectral intensities, the $b$ constant cancels out, leading to 2 equations with 3 unknowns $\left(a,\left\langle P_{2}\right\rangle\right.$ and $\left.\left\langle P_{4}\right\rangle\right)$.[29,36]

$$
\begin{aligned}
& R_{1}=\frac{I_{Z X}}{I_{Z Z}}=\frac{A\left\langle\left(\alpha_{Z X}\right)^{2}\right\rangle+B\left\langle\left(\alpha_{Z Y}\right)^{2}\right\rangle}{A\left\langle\left(\alpha_{Z Z}\right)^{2}\right\rangle+B\left\langle\left(\alpha_{Z Y}\right)^{2}\right\rangle} \\
& R_{2}=\frac{I_{X Z}}{I_{X X}}=\frac{A\left\langle\left(\alpha_{X Z}\right)^{2}\right\rangle+B\left\langle\left(\alpha_{X Y}\right)^{2}\right\rangle}{A\left\langle\left(\alpha_{X X}\right)^{2}\right\rangle+B\left\langle\left(\alpha_{X Y}\right)^{2}\right\rangle}
\end{aligned}
$$

The $A$ and $B$ constants enable taking into account the out-of-plane contributions to the measured signal intensity caused by the use of an objective with a high numerical aperture.[29,36,37] An equation is thus missing to solve the system of Eq. 4. The "traditional" method requires measuring the depolarization ratio $(\rho)$ on a perfectly isotropic $\left(\left\langle P_{2}\right\rangle=\left\langle P_{4}\right\rangle=0\right)$ sample with the same chemical and phase composition as the oriented sample of interest in order to extract the $a$ parameter (Eq. 5).[34,36] It is thus based on the 
approximation that the shape of the Raman tensor does not change upon orientation, an approximation that has been severely questioned by us and other groups.[31,32,38,39] With this method, called DC for "depol constant", the orientation parameters can thus be determined by solving Eqs. 4 and 5 .

$\rho=R_{1}=R_{2}=\frac{I_{Z X}}{I_{Z Z}}=\frac{I_{X Z}}{I_{X X}}=\frac{(A+B)(1-a)^{2}}{A\left(8 a^{2}+4 a+3\right)+B(1-a)^{2}}$

The recently developed "most probable distribution" (MPD) method eliminates the requirement of measuring the depolarization ratio (Eq. 5).[31,32] Instead, it makes the assumption that the orientation distribution is the most probable one and replaces Eq. 5 with the equation describing the most probable $\left\langle P_{4}\right\rangle\left(\left\langle P_{4}\right\rangle_{m p}\right)$ associated with a given $\left\langle P_{2}\right\rangle$ value (Eq. $6 \mathrm{a}$ and $6 \mathrm{~b}$ for parallel and perpendicular orientation distributions, respectively). The MPD method thus allows determining directly the $\left\langle P_{2}\right\rangle$ value without requiring further measurements on an isotropic sample, with use of Eqs. 4 and the appropriate Eq. 6.[40,41] It is noteworthy that the MPD method provides accurate $\left\langle P_{2}\right\rangle$ values even for samples associated with orientation distribution relatively different from the most probable one.[31]

$$
\begin{aligned}
& \left\langle P_{4}\right\rangle_{m p}=-0.083\left\langle P_{2}\right\rangle+1.366\left\langle P_{2}\right\rangle^{2}-1.899\left\langle P_{2}\right\rangle^{3}+1.616\left\langle P_{2}\right\rangle^{4} \\
& \left\langle P_{4}\right\rangle_{m p}=0.052\left\langle P_{2}\right\rangle+1.574\left\langle P_{2}\right\rangle^{2}+3.968\left\langle P_{2}\right\rangle^{3}+8.058\left\langle P_{2}\right\rangle^{4}
\end{aligned}
$$

\section{Results and discussion.}

Figure 2 illustrates the three most common types of collector used in the electrospinning field: a simple metallic surface (A), a "2 rods" collector (D) in which two 
conducting rods are separated by a short gap, and a rotating disk/drum (G). In all cases, the fibers were produced by applying a high voltage on the syringe containing the entangled solution of PEO dissolved in methanol (a volatile solvent). Once the solution jet is ejected from the spinneret due to electrostatic repulsion, it first follows a straight trajectory before entering the "whipping" region where it bends, causing a looping trajectory that takes the form of a cone.[42] The jet solidifies into a PEO fiber while being submitted to extremely high elongational forces that promote the significant reduction of its size to the nanometer scale, which in turn further increases the rate of solvent evaporation.[43] Solid fibers typically containing $2-5 \% \mathrm{w} / \mathrm{w}$ of residual solvent are then deposited on one of the three types of collector. The SEM of Figure 2B shows that when using an aluminum foil as the collector, the PEO fibers deposit as a macroscopically random network. After the positively charged jet adsorbs at the first contact point on the negatively charged collector, its selfrepulsion encourages the following contacts with the substrate to occur further away in a more or less random fashion. The two dimensional WAXD pattern (Figure 2C) of this random mat shows that PEO is highly crystalline due to the absence of an amorphous halo. The diffraction peaks are consistent with the normal monoclinic crystalline structure composed of chains with a $7 / 2$ helical conformation (composed of sequences of transtrans-gauche conformations[44,45]) as previously observed for PEO electrospun fibers.[13] All diffraction peaks appear as full circles along the azimuthal angle, indicating the absence of orientation of the crystals at the scale of the X-ray beam. In contrast, a preferential orientation would have led to arcs of circles with an azimuthal width inversely proportional to the orientation of the sample. 
Figure 2E shows a SEM picture of PEO fibers collected using two metallic rods as collector (Figure 2D). It shows a high level of alignment of the fibers but also the presence of defects such as fused fibers and beads that are detrimental to a perfect macroscopic organization. In this context, the positively charged jet first makes contact with one of the negatively charged rods and is then propelled towards the other rod, and so on, leading to a high level of alignment of the fibers between the rods. The 2D WAXD pattern of this sample (Figure 2F) shows azimuthal arcs of circles that are associated with the same crystalline phase as in Figure $2 \mathrm{C}$ and that reveal a clear preferential orientation along the fiber axis. It is possible to quantify the order parameter of the crystalline phase by WAXD using the azimuthal intensity distribution for a given diffraction plane.[46] The quantified $\left\langle P_{2}\right\rangle$ was 0.79 for this specific sample, a value in agreement with the infrared spectroscopy dichroic ratios reported by Kakade et al. for bundles of PEO fibers collected with a 2 rods collector [13] and also with those measured by WAXD on bundles of fibers of complexes of PEO with urea or thiourea.[46-48] Kakade et al.[13] proposed that at a molecular level, with this collection mode, the high polymer chains orientation is not due to the electrospinning process only but also to the reorientation of the solvent dipoles, caused by the additional electric field induced between the two negatively charged rods, that prevents the polymer chain relaxation.

[Figure 2 should be here] 
Figure $2 \mathrm{G}$ illustrates the last collection mode investigated, the rotating disk, which is probably the most popular one in its various implementations. In this case, the metallic disk rotates at high speed in order to promote a high level of fiber alignment. The alignment increases with the linear velocity of the collector until it matches the spinning rate, while a too high rotation speed leads to fiber breakage.[24,25] For a long time, orientation in electrospun fibers was believed to be exclusively induced by the mechanical stretching brought by the collector rotation at the final stage of the fiber formation, i.e. when the amount of residual solvent is small and thus when the relaxation of polymer chains is greatly reduced. As shown in Figure $2 \mathrm{H}$, the level of alignment is slightly lower and the density of defects is higher for PEO fibers collected with this method as compared with the 2 rods collector (Figure 2E). Consequently, the 2D WAXD pattern in Figure 2I shows broader diffraction arcs associated with a smaller $\left\langle P_{2}\right\rangle$ value of 0.60 . Kakade et al.[13] have also reported that electrospinning PEO fibers from an aqueous solution on a rotating collector produces bundles with a lower orientation than on a 2 rods collector, although the orientation was much lower in their experiments. In contrast, Kongkhlang et al.[14] have observed a very high orientation for polyoxymethylene (POM) nanofibers (a polymer that can reach very high crystallinity degrees like PEO) that they attributed to the additional mechanical stretching brought by the rotating collector.

The results of Figure 2 expose the large differences in the apparent $\left\langle P_{2}\right\rangle$ values for PEO fibers electrospun using different collectors but they also highlight the challenge of discriminating the real effects of the collector on molecular structure due to large differences in organization at the macroscopic scale (with a beam size of $\sim 400 \mu \mathrm{m}$ for our 
X-ray experiment). Accordingly, single fiber measurements were conducted by polarized confocal Raman spectroscopy to help discriminating these effects.

Figure 3A shows a typical set of the 4 polarized spectra required for orientation quantification on a PEO fiber of $\sim 700 \mathrm{~nm}$ diameter (inset of Figure $3 \mathrm{~A}$ ) collected with a 2 rods collector. The spectra were acquired following the protocol developed using poly(ethylene terephthalate) (PET) electrospun fibers as a model system.[29] Although PEO is a weakly scattering polymer and the fiber size is very close to the diffraction limit (in the context of our experiments, the diffraction-limited diameter of the laser spot focused on the sample is about $1 \mu \mathrm{m}$ ), polarized spectra with a very high signal to noise ratio can be obtained. The presence of some bands only in the ZZ or XX spectra highlights the very high preferential orientation of vibrational modes that are oriented along ( $\mathrm{Z}$ direction) or perpendicular (X direction) to the fiber axis and thus a very large $\left\langle P_{2}\right\rangle$ for this individual PEO fiber. The spectra are highly repeatable since the intensities are reproducible within 5 $\%$ when measuring a subsequent set of spectra on the same fiber, strongly supporting the absence of signal drift during the experiment that would be detrimental to the orientation quantification.

Figure 3B highlights some of the major features of the parallel-polarized spectra. For orientation purposes, we are particularly interested in the $1072 \mathrm{~cm}^{-1}$ band due to $\mathrm{CH}_{2}$ rocking and $\mathrm{COC}$ symmetric stretching for sequences of trans-gauche-trans conformations, [49] the $1230 \mathrm{~cm}^{-1}$ band associated with $\mathrm{CH}_{2}$ torsion in trans-trans-trans, trans-trans-gauche and trans-gauche-trans sequences in the helical conformation[49] and the $1386 \mathrm{~cm}^{-1}$ band associated with an out-of-phase $\mathrm{CH}_{2}$ wagging mode.[50] The presence of these bands mainly in one of the parallel-polarized spectra facilitates the orientation 
quantification because it allows concluding that the tilt angle of the Raman tensor associated to these modes is close to either 0 or $90^{\circ}$, without further investigations.

The $1072 \mathrm{~cm}^{-1}$ band was selected to quantify the orientation mainly because it is both well isolated and intense. An average $\left\langle P_{2}\right\rangle$ value of 0.88 was quantified on 15 different electrospun fibers of approximately the same diameter produced with the 2 rods collector (see Table 1) by applying the newly developed MPD method (i.e. by using only the four polarized spectra for each fiber, without the need to determine the depolarization ratio with an isotropic sample). This very large value is close to the theoretical maximum of 1 for a perfect orientation along the fiber axis and slightly higher than the one quantified by WAXD for bundles of fibers. It is worth noticing that the orientation values of individual fibers are expected to be higher due to the almost perfect alignment of a single fiber with respect to the incident laser polarization as compared to bundles of fibers which can never show a perfect macroscopic organization. The small $\left\langle P_{2}\right\rangle$ standard deviation of 0.06 also reveals the similitude of the characteristics from fiber to fiber. This result strongly contrasts with the very broad variations that were observed for PET[29] and polystyrene[30] electrospun fibers and might be explained by the highly crystalline nature of PEO (vide infra).

[Figure 3 should be here]

The shoulder appearing at $811 \mathrm{~cm}^{-1}$ and the band at $936 \mathrm{~cm}^{-1}$ are also identified in the spectra of Figure 3B. They have been associated by Koenig and Angood to the 
amorphous phase in their study of low molecular weight PEO in solution.[51] The presence of the $811 \mathrm{~cm}^{-1}$ shoulder mainly in the XX spectrum and the clear presence of the $936 \mathrm{~cm}^{-}$ ${ }^{1}$ band only in the $\mathrm{ZZ}$ spectrum suggest a preferential orientation of the amorphous phase in these fibers. The spectra also enable noticing the absence of bands at 1041 and $1151 \mathrm{~cm}^{-}$ ${ }^{1}$ which are observed when a polymorphic crystalline phase of PEO, where the chains adopt a planar zig-zag all-trans conformation, is formed under tension.[49] It is noteworthy that the absence of these bands does not imply that this polymorph cannot form under the extreme stretching conditions in the jet, but that in that case it would have relaxed (through a solid-solid transition) during the process.

Surprisingly, the polarized spectra recorded for fibers collected on the aluminum foil (see an example in Figure S1 of the Supporting Information) are almost identical to those shown in Figure 3. The quantification of orientation (on 15 fibers) thus led to an extremely similar average $\left\langle P_{2}\right\rangle$ value of $0.85 \pm 0.06$. Similarly, fibers collected with the rotating disk showed an identical orientation value of $0.86 \pm 0.08$. Accordingly, we conclude that the orientation of the crystals, when studied at the single fiber level, is extremely similar for all three collectors. The apparent differences in the WAXD results of Figure 2 therefore originate from differences in macroscopic alignment created by the collectors which have no impact on the residual orientation of PEO fibers.

For comparison, the $\left\langle P_{2}\right\rangle$ values were also quantified using the conventional DC method, which requires the additional measurement of 4 polarized spectra on an isotropic sample in order to extract the depolarization ratio associated with the band. A challenge with polymers such as PEO is the difficulty of producing a perfectly isotropic sample on the micron scale due to the large size of the spherulites formed during the crystallization 
process. We have shown by numerical simulations that small errors in the quantification of the depolarization ratio can, depending on its specific value, lead to relatively large errors on $\left\langle P_{2}\right\rangle$.[31] In the context of this study, we have estimated that $\rho=0.2$ for the $1072 \mathrm{~cm}^{-1}$ band based on measurements on an imperfectly isotropic sample. It is noteworthy that even when a perfectly isotropic sample is available, the evolution of $\rho$ upon orientation can lead to erroneous orientation values, especially when it is small as here.[32]

The $\left\langle P_{2}\right\rangle$ values reported in Table 1 are higher when quantified with the DC method than with the MPD method and their larger standard deviations clearly illustrate that the DC method often leads to values beyond the theoretical maximum of 1 . These larger standard deviations, in spite of the fact that the calculations were done using the same polarized spectra, are most likely due to the significant fluctuation of the $a$ parameter (see Eq. 5) for small variation of $\rho$ in the range investigated.[31] Overall, this comparison illustrates, that in addition to its experimental simplicity, the MPD method provides a better accuracy than the DC method. However, despite the fact that some of the values quantified by the DC method are questionable, it is noteworthy that they lead to the same main conclusion: an absence on any collector effect on orientation in individual PEO fibers.

\section{[Table 1 should be here]}

The results of Table 1 suggest that the orientation of the fibers was frozen in before the jet reached the collector such that mechanical stretching or additional electric field effects could not modify the organization of the chains. This conclusion is in contradiction 
with most of the studies that have investigated the impact of the collector on molecular orientation, especially those performed on bundles of fibers collected on a rotating disk.[12,24,25,52-54] It is also at odds with SAED studies of individual fibers of poly(vinylidene fluoride) (PVDF)[23,26] and poly [(R)-3-hydroxybutyrate-co(R)-3hydroxyhexanoate] (PHBHx)[55] where it was shown that small changes of collector parameters can cause significant differences on the level of molecular orientation. Generally, the reduction of the fiber diameter under certain collection conditions is considered to be a direct proof of supplementary stretching that can justify the higher residual orientation. In this study, no significant changes in diameter distribution were observed from the SEM images, although we observed the formation of a few very large ribbon fibers in all conditions. Additionally, although this factor has not been systematically studied here, the diameter of the fibers does not appear to play a significant role since PEO fibers with a diameter of several microns (produced from an acetone solution) led to spectra extremely similar to those of Figure 3 (see Figure S2 of the Supporting Information) and thus to similar $\left\langle P_{2}\right\rangle$ values.

We believe the justification for this absence of collector effect resides in the specific characteristics of this polymer. PEO has an extremely low glass transition temperature of $\sim-60{ }^{\circ} \mathrm{C}[56]$ that enables chain mobility and orientation relaxation even when the fraction of residual solvent is low. However, it is also characterized by its capacity to reach extremely high crystallinity degrees due to its very fast crystallization kinetics as compared to semi-crystalline polymers, as observed in our WAXD results. In this context, we propose that the stretching of the chains in the jet favors the rapid formation of highly directionalized nuclei that rapidly grow to form larger fibrillar crystals, such that the 
orientation is driven by the crystallization process in these fibers. Once formed, the crystals orientation relaxation time is extremely high as compared to that of loose amorphous polymer chains and the high crystallinity degree reached prior to the deposition on the collector hinders any further stretching. We thus expect that the conclusions drawn here should apply to fibers composed of other polymers with very fast crystallization rates such as POM and polyethylene, but not for amorphous or weakly crystalline polymers. Overall, they expose the necessity of thoroughly studying and revisiting our comprehension of the impact of several electrospinning parameters on the characteristics of individual fibers composed of different types of polymers.

\section{Conclusion.}

In this publication, we have investigated the effect of the collector on the molecular orientation of PEO electrospun fibers deposited on a metallic plate, a 2 rods collector and a rotating disk. Confocal Raman spectroscopy on individual fibers of typical diameter of $700 \mathrm{~nm}$ has highlighted the extremely high level of molecular orientation reached in PEO fibers as well as the applicability of our recently developed MPD orientation quantification methodology as compared to the conventional DC method. No noticeable effect of the collector was found, in disagreement with the WAXD results on bundles of the same fibers and with previous studies performed on bundles of fibers composed of PEO and other polymers. We have established that, at least in the context of PEO, this discrepancy is due to the relative alignment of the fibers when studied as bundles. We propose that the orientation is mainly driven by the fast and efficient crystallization process of PEO which 
happens at an earlier stage of the fiber formation process, therefore limiting relaxation and preventing any further stretching that could be induced by the collector. This study highlights the importance and the advantages of studying the impact of electrospinning parameters on the structure of electrospun fibers at the individual fiber scale using simple and efficient spectroscopic techniques in order to draw accurate and clear conclusions.

\section{Acknowledgments.}

This work was supported by a grant and a graduate scholarship (MRL) from the Natural Sciences and Engineering Research Council of Canada (NSERC). We are grateful to A. Gittsegrad for the SEM imaging.

\section{References.}

[1] S. Agarwal, J.H. Wendorff, A. Greiner, Adv. Mater. 21 (2009) 3343-3351.

[2] T.J. Sill, H.A. von Recum, Biomaterials 29 (2008) 1989-2006.

[3] D.R. Nisbet, J.S. Forsythe, W. Shen, D.I. Finkelstein, M.K. Horne, J. Biomater. Appl. 24 (2009) 7-29.

[4] W.J. Li, C.T. Laurencin, E.J. Caterson, R.S. Tuan, F.K. Ko, J. Biomed. Mater. Res. 60 (2002) 613-621.

[5] W. Cui, Y. Zhou, J. Chang, Sci. Technol. Adv. Mat. 11 (2010) 014108.

[6] D. Tu, S. Pagliara, A. Camposeo, L. Persano, R. Cingolani, D. Pisignano, Nanoscale 2 (2010) 2217-2222.

[7] Y. Xin, Z. Ling, S. Li, T. Lin, G. Liu, Mater. Sci. Eng. B-Adv. 177 (2012) 1094-1097.

[8] J.-Y. Chen, C.-C. Kuo, C.-S. Lai, W.-C. Chen, H.-L. Chen, Macromolecules 44 (2011) 2883-2892.

[9] J.M. Moran-Mirabal, J.D. Slinker, J.A. DeFranco, S.S. Verbridge, R. Ilic, S. FloresTorres, H. Abruña, G.G. Malliaras, H. Craighead, Nano letters 7 (2007) 458-463.

[10] V. Fasano, M. Moffa, A. Camposeo, L. Persano, D. Pisignano, Macromolecules 48 (2015) 7803-7809.

[11] M. Richard-Lacroix, C. Pellerin, Macromolecules 46 (2013) 9473-9493.

[12] S.F. Fennessey, R.J. Farris, Polymer 45 (2004) 4217-4225.

[13] M.V. Kakade, S. Givens, K. Gardner, K.H. Lee, D.B. Chase, J.F. Rabolt, J. Am. Chem. Soc. 129 (2007) 2777-2782.

[14] T. Kongkhlang, K. Tashiro, M. Kotaki, S. Chirachanchai, J. Am. Chem. Soc. 130 (2008) 15460-15466. 
[15] P. Katta, M. Alessandro, R.D. Ramsier, G.G. Chase, Nano Letters 4 (2004) 22152218.

[16] L.S. Carnell, E.J. Siochi, N.M. Holloway, R.M. Stephens, C. Rhim, L.E. Niklason, R.L. Clark, Macromolecules 41 (2008) 5345-5349.

[17] D. Li, G. Ouyang, J.T. McCann, Y. Xia, Nano Letters 5 (2005) 913-916.

[18] H. Li, Y. Xu, H. Xu, J. Chang, J. Mater. Chem. B 2 (2014) 5492-5510.

[19] J. Xie, W. Liu, M.R. MacEwan, P.C. Bridgman, Y. Xia, ACS Nano 8 (2014) 18781885.

[20] J. Joseph, S.V. Nair, D. Menon, Nano Letters 15 (2015) 5420-5426.

[21] G.S. Bisht, G. Canton, A. Mirsepassi, L. Kulinsky, S. Oh, D. Dunn-Rankin, M.J. Madou, Nano Letters 11 (2011) 1831-1837.

[22] Y. Ner, C. Asemota, J.R. Olson, G.A. Sotzing, ACS Appl. Mater. Interfaces 1 (2009) 2093-2097.

[23] X. Ma, J. Liu, C. Ni, D.C. Martin, B.D. Chase, J.F. Rabolt, J. Polym. Sci. B: Polym. Phys. 54 (2016) 617-623.

[24] M.D. Edwards, G.R. Mitchell, S.D. Mohan, R.H. Olley, Eur. Polym. J. 46 (2010) $1175-1183$.

[25] S.D. Mohan, G.R. Mitchell, F.J. Davis, Soft Matter 7 (2011) 4397.

[26] X. Ma, J. Liu, C. Ni, D.C. Martin, D.B. Chase, J.F. Rabolt, ACS Macro Letters 1 (2012) 428-431.

[27] T. Yoshioka, R. Dersch, A. Greiner, M. Tsuji, A.K. Schaper, Macromol. Mater. Eng. 295 (2010) 1082-1089.

[28] T. Yoshioka, R. Dersch, M. Tsuji, A.K. Schaper, Polymer 51 (2010) 2383-2389.

[29] M. Richard-Lacroix, C. Pellerin, Macromolecules 45 (2012) 1946-1953.

[30] M. Richard-Lacroix, C. Pellerin, Macromolecules 48 (2015) 4511-4519.

[31] M. Richard-Lacroix, C. Pellerin, Appl. Spectrosc. 67 (2013) 409-419.

[32] M. Richard-Lacroix, C. Pellerin, Macromolecules 46 (2013) 5561-5569.

[33] I.M. Ward, Structure and Properties of Oriented Polymers, 2nd Edition, Chapman \& Hall, London, 1997.

[34] T. Lefèvre, C. Pellerin, M. Pézolet, Characterization of Molecular Orientation, in: J.M. Chalmers, R.J. Meier (Eds.) Comprehensive Analytical Chemistry, Elsevier, 2008, pp. 295-335.

[35] D.I. Bower, J. Polym. Sci. A-2: Polym. Phys. 10 (1972) 2135-2153.

[36] M.E. Rousseau, T. Lefèvre, L. Beaulieu, T. Asakura, M. Pézolet, Biomacromolecules 5 (2004) 2247-2257.

[37] G. Turrell, J. Raman Spectros. 15 (1984) 103-108.

[38] S. Frisk, R.M. Ikeda, D.B. Chase, J.F. Rabolt, Appl. Spectrosc. 57 (2003) 1053-1057.

[39] S. Yang, S. Michielsen, Macromolecules 36 (2003) 6484-6492.

[40] H. Pottel, W. Herreman, B.W. van der Meer, M. Ameloot, Chem. Phys. 102 (1986) 37-44.

[41] F. Lagugné-Labarthet, T. Buffeteau, C. Sourisseau, Appl. Spectros. 54 (2000) 699705.

[42] Y.M. Shin, M.M. Hohman, M.P. Brenner, G.C. Rutledge, Appl. Phys. Lett. 78 (2001) $1149-1151$. 
[43] D.H. Reneker, A.L. Yarin, E. Zussman, H. Xu, Electrospinning of Nanofibers from Polymer Solutions and Melts, in: H. Aref, E. van der Giessen (Eds.) Advances in Applied Mechanics, Elsevier, 2007, pp. 43-346.

[44] Y. Takahashi, H. Tadokoro, Macromolecules 6 (1973) 672-675.

[45] H. Tadokoro, Y. Chatani, T. Yoshihara, S. Tahara, S. Murahashi, Die Makromolekulare Chemie 73 (1964) 109-127.

[46] Y. Liu, C. Pellerin, Macromolecules 39 (2006) 8886-8888.

[47] Y. Liu, H. Antaya, C. Pellerin, J. Polym. Sci. B 46 (2008) 1903-1913.

[48] Y. Liu, H. Antaya, C. Pellerin, J. Phys. Chem. B 114 (2010) 2373-2378.

[49] Y. Ding, J.F. Rabolt, Y. Chen, K.L. Olson, G.L. Baker, Macromolecules 35 (2002) 3914-3920.

[50] T. Yoshihara, H. Tadokoro, S. Murahashi, J. Chem. Phys. 41 (1964) 2902-2911.

[51] J.L. Koenig, A.C. Angood, J. Polym. Sci. A-2: Polym. Phys. 8 (1970) 1787-1796.

[52] A. Baji, Y.-W. Mai, S.-C. Wong, M. Abtahi, P. Chen, Comp. Sci. Technol. 70 (2010) 703-718.

[53] W.A. Yee, M. Kotaki, Y. Liu, X. Lu, Polymer 48 (2007) 512-521.

[54] W.A. Yee, A.C. Nguyen, P.S. Lee, M. Kotaki, Y. Liu, B.T. Tan, S. Mhaisalkar, X. Lu, Polymer 49 (2008) 4196-4203.

[55] L. Gong, D.B. Chase, I. Noda, J. Liu, D.C. Martin, C. Ni, J.F. Rabolt, Macromolecules 48 (2015) 6197-6205.

[56] J.F. Le Nest, S. Callens, A. Gandini, M. Armand, Electrochim. Acta 37 (1992) 15851588 .

Table 1. Orientation parameters $\left(\left\langle P_{2}\right\rangle\right)$ quantified by Raman spectroscopy on individual PEO fibers (with the MPD and DC methods) and by WAXD on fiber bundles, using the 3 collection modes indicated.

\begin{tabular}{lcc|c}
\hline & \multicolumn{2}{c|}{ Raman $^{*}$} & WAXD $^{* *}$ \\
\cline { 2 - 4 } & $\left\langle P_{2}\right\rangle_{\text {MPD }}$ & $\left\langle P_{2}\right\rangle_{\mathbf{D C}}$ & $\left\langle P_{2}\right\rangle_{\mathbf{1 2 0}}$ \\
\cline { 2 - 4 } Metallic plate & $0.85 \pm 0.06$ & $0.92 \pm 0.14$ & 0 \\
2 rods & $0.88 \pm 0.05$ & $0.95 \pm 0.12$ & $0.73 \pm 0.06$ \\
Rotating disk & $0.86 \pm 0.08$ & $0.96 \pm 0.09$ & $0.61 \pm 0.02$ \\
\hline
\end{tabular}

* Averaged over 15 different fibers from independent samples.

** Averaged over 3 independent samples. 


\section{Figure captions.}

Figure 1. Schematic representation of a typical Raman spectroscopy experiment for orientation quantification of different phases in a single fiber. The polarization of the incident laser and of the scattered light are fixed parallel $(\mathrm{Z})$ or perpendicular $(\mathrm{X})$ to the fiber axis with a half-wave plate $(\lambda / 2)$ and an analyzer, respectively, and $\mathrm{Y}$ is the light propagation direction. The bottom sketches illustrate two possible cases for the orientation of a fiber composed of a semi-crystalline polymer (dark crystallites and light amorphous chains) that can be discriminated by the measurement of the $\left\langle P_{2}\right\rangle$ value.

Figure 2. Schematic representation of the electrospinning setup, SEM picture showing the level of fiber alignment, and 2D WAXD pattern for fibers collected with A-C) a metallic plate collector, D-F) a 2 rods collector, and G-I) a rotating disk.

Figure 3. A) Representative set of the four polarized spectra required for orientation quantification by Raman spectroscopy measured on a PEO fiber of $700 \mathrm{~nm}$ diameter (inset) collected with a 2 rods collector. B) Zoom of the parallel-polarized spectra of the same fiber. 